\title{
Influence of Feedback on Learning
}

\author{
SAHIBA KUKREJA ${ }^{1}$ AND TeJINDER SINGH ${ }^{2}$ \\ From the Departments of ${ }^{1}$ Biochemistry, ${ }^{2}$ Pediatrics, and ${ }^{1,2}$ Medical Education; Sri Guru Ram Das Institute of Medical Sciences and \\ Research, Amritsar, Punjab, India.drtejinder.sgrd@gmail.com
}

$\mathrm{T}$ he advent of newer learner-focused methodologies in medical education, such as problem-based learning, competency-based medical education and self-directed learning, has brought the focus on feedback as an important tool to improve learning. Feedback is believed to improve learning by reducing the discrepancy between the desired and actual understanding. In general educational settings, Hattie and Timperley [1] reported the most comprehensive synthesis of over 500 meta-analyses, involving hundreds of thousands of studies. The effect size of feedback was 0.79 amongst hundreds of factors that could influence achievements. A similar analysis was reported by Veloski, et al. [2] in medical education. Of the studies included, 74\% demonstrated that feedback alone had a positive influence on learning. However, the effect size in medical education was less as compared to general education, possibly related to complexity of tasks. To be effective, feedback has to be based on direct observation of the learner - using tools like Objective Structured Clinical Examination (OSCE), Direct Observation of Procedural Skills (DOPS) and Mini-Clinical Evaluation Exercise (mini-CEX) [3-5]. The Hattie and Timperley review also brought into focus the 'type' of feedback and its influence on achievement. It is interesting to see that with even small changes in the way feedback is delivered, the effect sizes can vary. For example, the reported differences between 'performance is correct $v s$ incorrect' (0.43 vs 0.25), 'discouraging the learner vs not doing so' (-0.14 vs 0.33$)$, 'praise vs no praise' (0.09 vs 0.34$)$ and 'complex vs non-complex task' (0.03 vs 0.55$)$ [6] only highlight the fact that giving feedback is much more than simply telling something to the learner. In addition, effectiveness of feedback depends on its successful acceptance by the learners [7]. This means that some types of feedback would be more effective as compared to others, and it is therefore complex to quantify the effect in most situations. Kulhavy [8] suggests that feedback and instructions should be seen as two ends of the same continuum, meaning that the process itself takes on the form of new instruction, rather than informing the learner solely about correctness. Lastly, not only the teachers need to perfect the art (and science) of giving feedback, the learners also need to be prepared to receive feedback and use it effectively [9].

This issue of Indian Pediatrics carries an article by Al Khateeb, et al. [10] examining the role of a single formative OSCE on subsequent summative performance. The authors divided the class into intervention and control groups, out of which the intervention group was given feedback following a formative OSCE on competencies acquired during previous modules. However, this group did not better its performance in the subsequent summative OSCE, while control group did. Apparently, the results seem to be counter to the widely held educational belief that feedback improves performance. Some of the possible reasons for this provided by the authors include the use of marks/grades for feedback, delay in providing feedback, and more practice in taking summative OSCE by the control group. However, the major issue with this study seems to be that formative OSCE was only a one time event, and that it related to the competencies acquired in the previous modules and not to those which were being acquired. Similarly, the quality and type of feedback may not have been standardized. Moreover, a single instance of feedback may not really have the desired effect. It is also to be noted that while the intervention group may have lost some time in the formative OSCE exercise, the control group worked on the modular teaching for that phase. Since details about the competencies tested by OSCE are not provided, it is difficult to comment on the immediate versus delayed feedback.

Despite some of its limitations, this paper does carry some important messages for us. The first relates to training the teachers and learners alike to give and receive feedback. Variations in the quality and type of feedback can negate all the benefits of such an exercise. Secondly, feedback should not be seen as a one time intervention. Rather, it should be an ongoing process, with focus on the tasks being learnt. Lastly, marks are not the true 
reflection of learning. They can be influenced by a number of factors, including variations in sampling and the difficulty level of tests. This paper also tells us that feedback as a single one time activity may not result in improvement. Preparing both teachers and learners is also important for feedback to be successful.

Lest we get some contradictory messages, instead of asking 'should we give feedback', a better question to ask will be 'how should we give feedback.' The utility of continuous, ongoing, authentic feedback based on direct observation of performance and given in an atmosphere of trust and in a non-threatening environment has never been doubtful!

Funding: None Conflict of interest: None stated.

\section{REFERENCES}

1. Hattie J, Timperley H. The power of feedback. Rev Educ Res. 2007;77:81-112.

2. Veloski J, Boex JR, Grasberger MJ, Evans A, Wolfson DB. Systematic review of the literature on assessment, feedback and physicians' clinical performance. Med Teach. 2006;28:117-28.
3. Norcini JJ. The power of feedback. Med Educ. 2010;44: 16-7.

4. Khalil S, Aggarwal A, Mishra D. Implementation of a miniclinical evaluation exercise (mini-CEX) program to assess the clinical competence of postgraduate trainees in Pediatrics. Indian Pediatr. 2017;54:284-7.

5. Singh T, Kundra S, Gupta P. Direct observation and focused feedback for clinical skills training. Indian Pediatr. 2014;51:713-7.

6. Kluger AN, Denisi A. The effects of feedback interventions on performance: A historical review, a meta-analysis, and a preliminary feedback intervention theory. Psychol Bull. 1996;119:254-84.

7. Ende J. Feedback in clinical medical education. JAMA. 1983;250:777-81.

8. Kulhavy RW. Feedback in written instruction. Rev Educ Res. 1977;47:211-32.

9. Delva D, Sargeant J, Miller S, Holland J, Brown PA, Leblanc C, et al. Encouraging residents to seek feedback. Med Teach. 2013;35:e1625-31.

10. Alkhateeb NE, Al-Dabbagh A, Ibrahim M, Al-Tawil NG. Effect of a formative objective structured clinical examination on the clinical performance of undergraduate medical students in a summative examination: A randomized controlled trial. 2019;56:745-8. 